\title{
Modelbase System in Remote Sensing Information Analysis and Service Grid Node
}

\author{
Yong Xue $^{1,2}$, Lei Zheng ${ }^{1,3}$, Ying Luo ${ }^{1,3}$, Jianping Guo ${ }^{1,3}$, Wei Wan ${ }^{1,3}$, Wei Wei ${ }^{1,3}$, \\ and Ying Wang ${ }^{1,3}$
}

\footnotetext{
${ }^{1}$ State Key Laboratory of Remote Sensing Science, Jointly Sponsored by the Institute of Remote Sensing Applications of Chinese Academy of Sciences and Beijing Normal University, Institute of Remote Sensing Applications, Chinese Academy of Sciences, P.O. Box 9718, Beijing 100101, China

${ }^{2}$ Department of Computing, London Metropolitan University, 166-220 Holloway Road, London N7 8DB, UK

${ }^{3}$ Graduate School, Chinese Academy of Sciences, Beijing 100049, China y.xue@londonmet.ac.uk
}

\begin{abstract}
In this article we describe a modelbase system used in Remote Sensing Information Analysis and Service Grid Node (RISN) at Institute of Remote Sensing Applications (IRSA), Chinese Academy of Sciences (CAS). The goal of the Node is to make good use of physically distributed resources in the field of remote sensing science such as data, models and algorithms, and computing resource left unused on Internet. With the modelbase system, we can organize and manage models better and make full use of models. With this system, we update both local and remote models easily, and we can also add remote modelbase into our modelbase management system. At the same time, we provide interfaces to access and run models from our node. Implementing it, we use Oracle to organize and manage models, use java language to connect with Oracle database and run models on Condor platform.
\end{abstract}

Keywords: Modelbase system, Remote sensing information analysis and Service Grid Node, modelbase management system.

\section{Introduction}

The research of model management theory began 80th last century. In 1980 Blanning (1980) first imported the notion of modelbase, and designed model query language (MQL) like database query language to management models. Geoffrion (1987) designed structural model language (SML), which introduced structural program design into building models. Muhanna et al. (1988) introduced system theory into modelbase management system. Wesseling et al. (1996) designed dynamic model language to support special data structural.

Modelbase can be devised into two kinds by its models: graph modelbase whose models are graph and arithmetic modelbase whose models are arithmetic or program. Kuntal et al. (1995) Organized Large Structural Modelbases which were full of graph 
models at 1995, which gave a good example of graph modelbase, but arithmetic modelbase is different according its application. Liu et al. built a simulation supporting system of cold rolling process control system based on modelbase. Li et al. (2002) built water environment models and put them in modelbase. So we present an arithmetic modelbase that is used in remote sensing application and also can connect to Grid environment - Condor platform through RISN at IRSA, CAS, China based on the Condor platform.

The node is a special node of Spatial Information Grid (SIG) in China. The node will be introduced in Section 2. The modelbase system and the function of modelbase in the node will be demonstrated in Section 3. Finally, the conclusion and further development will be addressed in Section 4.

\section{Remote Sensing Information Analysis and Service Grid Node}

Remotely sensed data is one of the most important spatial information sources, so the research on architectures and technical supports of RISN is the significant part of the research on SIG. The aim of the Node is to integrate data, traditional algorithm and software, and computing resource distributed, provide one-stop service to everyone on Internet, and make good use of everything pre-existing. The Node is extendable, which contains many personal computers, supercomputers or other nodes. It also can be as small as just on personal computer. There are two entries to the Node: 1) A portal implemented by JSP. User can visit it through Internet browses, such as Internet Explorer and others; 2) A portal implemented by web service and workflow technology. It is special for SIG. Other Grid resources and Grid systems can be integrated with our node through this portal. The node provides the application services such as aerosol optical depth retrieval, land surface temperature retrieval, soil moisture retrieval, surface reflectance retrieval, and vegetation index applications from MODIS data. To manage models, we used modelbase system in the node. Figure 1 shows the structure of our modelbase system.

\section{Modelbase System in Remote Sensing Information Analysis and Service Grid Node}

Modelbase system has two main parts: modelbase file system and modelbase management system.

\subsection{Modelbase File System}

Modelbase file system is the place where models are stored. In the RISN, models are stored in several computers, remote computers and remote modelbases. All models treated as virtually stored in one computer with modelbase management system. We achieve the distributed management system in this architecture. The system has another benefit: we can add both models separately or in remote modelbase freely but not download models to our computers. In our RISN, we provide Condor platform to run models on Grid system, so the architecture assumes to the option of sharing resources in the Grid computing. 


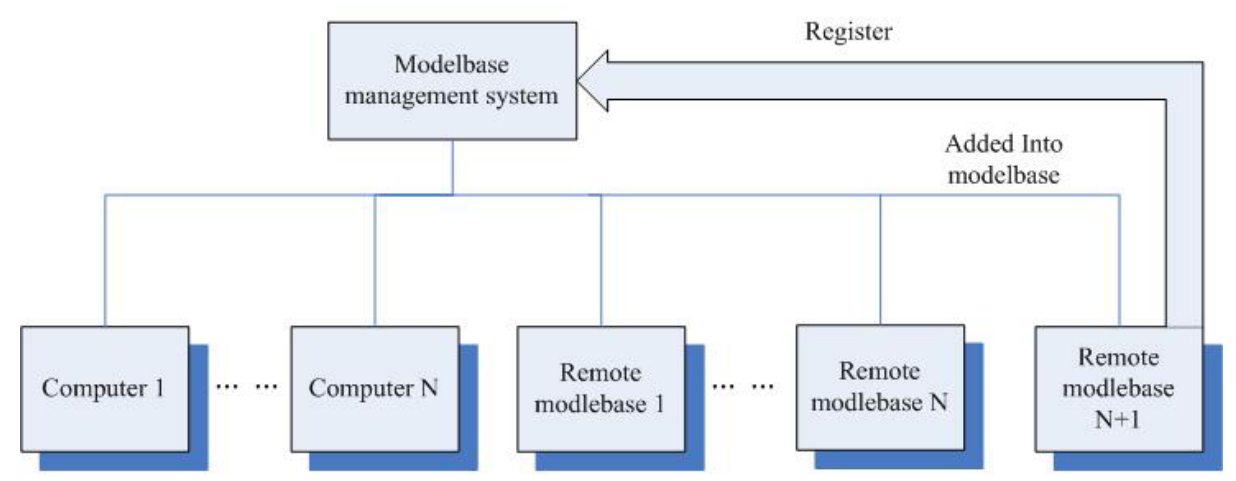

Fig. 1. Information registry of models in remote modelbase

\subsection{Modelbase Management System}

Modelbase management system contains the registry information of models, the interface with Condor platform and the interface with portal.

\subsubsection{Registry of Models}

We import notion of meta-module (Xue et al. 2006) and extends it in our system. In our system, every model is a meta-module, and the information is stored in the table of modelbase.

\subsubsection{Updating in Remote Modelbase}

In our design, we can add remote modelbase expediently but no need to download models to our computer. But in order to add models in remote computer into our modelbase system, the information of models is needed to be registered, and a method is needed to be provided to get models if you want to provide your models to run by others. If you register your modelbase information in our modelbase system, we will provide an interface to connect to your modelbase and make it part of our modelbase.

\subsubsection{Models Access and Running on a Grid Computing Platform}

As our modelbase system is one part of RISN, we provide interface to connect with our portal and workflow.

After select the model you want to run, you can run by pressing the "submit" button on the portal, or download model to your own computer according to the information of the model to run. If you choose to run the model on our Remote Sensing Information Analysis and Service Grid Node, we provide some model to run on the Condor platform, you need to input parameter as required.

\section{Conclusion and Future Work}

Grid technology is a very effective method for remotely sensed data processing service. Through it the existing remotely sensed models and algorithms resource can 
be shared seems like the common service in Grid environment. In this article we describe a modelbase system used in Remote Sensing Information Analysis and Service Grid Node which can be used on Condor platform. With the modelbase system, we can organize and manage models better and make full use of models and run models on Condor platform. Any models can be added by any users who are authorized, in the future, models will be added to modelbase only after checked by administrator.

\section{Acknowledgment}

This publication is an output from the research projects "Multi-scale Aerosol Optical Thickness Quantitative Retrieval from Remotely Sensing Data at Urban Area" (40671142) and "Grid platform based aerosol monitoring modeling using MODIS data and middlewares development" (40471091) funded by NSFC, China and "863 Program - Remote Sensing Information Processing and Service Node" funded by the MOST, China.

\section{References}

1. Blanning, R.W.,: How Managers Decide to Use Planning Models. Long Range Planning. 13(1980) 32-35

2. Geoffrion, A.,: An Introduction to Structured Modeling. Management Science. 33:5(1987)547-588

3. Muhanna, W.A., and Roger, A.P.: A System Framework for Model Software Management. TIMS/ORSA meeting, Washington, DC. 4(1988) 